\title{
EVALUACIÓN Y REDUCCIÓN DE LA VULNERABILIDAD: UN ENFOQUE INDISPENSABLE PARA LA GESTIÓN TERRITORIAL
}

\author{
POR \\ NELLY AMALIA GRAY DE CERDÁN
}

Según la OEA «desde 1960, los terremotos, huracanes, inundaciones, sequías, desertificación y deslizamientos de tierra en la región latinoamericana y del Caribe, han causado la muerte de más de 180.000 personas, han afectado la vida de 100 millones más y han causado más de 54 millones de dólares en daños a la propiedad. El monto de destrucción aumenta década tras década y los efectos adversos sobre el empleo, la balanza comercial y la deuda externa perduran por varios años después de un desastre». «Lo peor de todo es que los países más pobres y los sectores más pobres de sus respectivas poblaciones, son los que sufren los impactos más severos. La rehabilitación y la ayuda internacional compensa a los paísess afectados en sólo una pequeña parte de las pérdidas que sufren.» ${ }^{1}$

El Territorio ha sido siempre fuente de recursos y posibilidades para la organización de la vida del hombre. Las últimas décadas del si-

${ }^{1}$ O.E.A.: Manual sobre el manejo de peligros naturales en la planificación para el Desarrollo Regional Integrado. Departamento de Desarrollo Regional y Medio ambiente. Secretaría Ejecutiva para asuntos Económicos y Sociales de los Estados Americanos, Washington D.C., 1993, p. xi.

Nelly Amalia Gray de Cerdán. C.O.N.I.C.E.T. (Consejo de Investigaciones Científicas y Ténicas). C.E.T.E.M. (Centro de Estrategias Territoriales para el MERCOSUR). Departamento Geografía - Facultad de Filosofía y Letras, Universidad Nacional de Cuyo.

Estudios Geográficos

Tomo LVIV, n. ${ }^{\circ} 230$, enero-marzo, 1998 
glo ponen en evidencia, sin embargo, el despilfarro de recursos naturales que ha realizado el hombre al amparo de los regímenes económicos dominantes, la reducción alarmante del patrimonio natural disponible para las próximas generaciones, el daño producido a los ecosistemas y sobre todo el impacto que su accionar tiene sobre las posibilidades de supervivencia de la especie humna.

Hoy este accionar irresponsable se vuelve en contra de la humanidad, poniendo en peligro su vida diaria, su capacidad de producir, aumentando los costos de su instalación y haciéndola cada vez más vulnerable frente a la Naturaleza.

Se dispone de una extraordinaria masa de información que está permitiendo produndizar esta rereflexión, orientando a los habitantes de la Tierra y a avanzar en una nueva filosofía de organización, basada en procesos de globalización, integración y colaboración ya que la modificación sustancial en el patrimonio de recursos, la creciente vulnerabilidad y la necesidad de manejarse con criterios de sustentabilidad, ponen de manifiesto no sólo la necesidad de modificar las formas de producción y las relaciones entre el hombre y la naturaleza, sino sobre todo de un cambio en: la manera de administrar los recursos, que comienzan a ser escasos y en el modo de encarar la gestión global del territorio y su desarrollo.

Nuevo vocabulario para la Gestión Territorial: amenazas, vulnerabilidad y riesgo

Las estadísticas nos muestran que en los últimos años los desastres debidos a peligros naturales han crecido en forma alarmante. Sin embargo, las manifestaciones de los fenómenos físico-naturales no han cecido... ¿Por qué entonces se afirma que han aumentado los desastres?

Para comprender estos problemas hay que partir de definir ciertos términos que se incorporan como sinónimos en el análisis territorial pero que no son similares entre sí:

Amenazas.-Son los peligros que existen en torno a las comunidades humanas y que pueden crear situaciones adversas para la vida del hombre y el desarrollo de su actividad. Históricamente se ha hablado de ame- 
nazas (o peligros) naturales y amenazas tecnológicas. Los estudios más modernos las clasifican en amenazas naturales, socio-naturales, antrópico-contiminantes y antrópico-tecnológicas ${ }^{2}$.

- Las primeras son el fruto de la dinámica terrestre. De origen geotectónico, geodinámico, meteorológico, hidrológico, etc., producen sismos y deslizamientos, huracanes e inundaciones, sequías, etc. Son parte del funcionamiento del medio ambiental natural del ser humano y no admiten modificaciones ni control par parte del hombre.

- Las segundas - socio-naturales - son el el fruto de una mala intervención del hombre sobre la naturaleza en su afán de controlarla: inundaciones, deslizamientos, hundimientos, agotamiento de acuíferos, efecto invernadero, etc., suelen ser confundidos y considerados como amenazas naturales y por consiguiente, fenómenos sin control. Sin embargo, si modificamos la acción humana, es posible mitigar sus efectos; por ejemplo: la deforestación en la zona sur de Brasil han acentuado y creado inundaciones muy peligrosos en la Pampa Argentina.

- Las terceras - antrópico-contaminantes - también son fruto de una defectuososa intervención humana, pero se relaciona con los procesos de contaminación derivados de derrames, dispersiones o emisiones de sustancias químioc-tóxicas de las actividades que el hombre ha creado. Generalmente son producto de la negligencia y de la falta de controles sobre los procesos productivos: plaguicidas, gases tóxicos, eliminación de desecho sólidos, líquidos o gaseosos. En general, son muy frecuentes en las ciudades y su impacto aumenta en forma proporcional al del proceso de concentración humana.

- Las cuartas - antrópico-tecnológicas- están vinculadas sobre todo con la seguridad de los procesos tecnológicos y las formas de distribución y organización que el hombre a adoptado en este tema. Las fallas en esos procesos genera una serie insospechada de amenazas, que puede comprometer a veces regiones enteras o grandes extensiones del planeta.

${ }^{2}$ Lavell Alan: «Degradación ambiental, riesgo y desastre urbano. Problemas y conceptos: hacia la definición de una agenda de investigación». en Ciudad en Riesgo. La RED-USAID, Perú, 1996. 
Por ejemplo Chernobyl (planta nuclear), Bhopal (planta química) oleoductos de gasolina en Guadalajara (México), etc. Son amenazas que están vinculadas estrictamente con la acción humana.

Estos diferentes tipos de amenazas pueden presentarse en forma independiente o bien combinadas, creando veces situaciones de verdaderos desastres humanos cuando se producen. Pero indudablemente las amenazas que están en crecimiento son las tres últimas, es decir, aquellas que en mayor o menos medida dependen de la acción humana.

Por lo tanto, en un alto porcentaje, podemos mitigar sus efectos si actuamos en forma inteligente y previsora, adoptando formas de organización y de intervención que minimicen el riesgo.

Vulnerabilidad.-Se define como la propensión de sufrir daños que tiene un componente de la estructura social o de la naturaleza misma. Es la forma en que las comunidades se exponen voluntaria o inconscientemente a un peligro natural o humano. «En ese sentido, la vulmerabilidad es la expresión del desequilibrio o desajuste entre la estructura social y el medio físico constructivo y natural que nos rodea» ${ }^{3}$.

La instalación del hombre en laderas de los volcanes para aprovechar las buenas tierras para el cultivo, la instalación con alta densidades demográficas en zonas de alto peligro sísmico, el asentamiento de comunidades en riberas sometidas a procesos erosivos o en zonas de deslizamientos, etc., marcan una forma de organización humana que, movida generalmente por principios de oportunidad económica o tradiciones históricas, convierten a hombre en una potencial víctima de la naturaleza

La vulnerabilidad depende, en líneas general, del tipo e intensidad de la amenaza; en consecuencia, no tiene un valor absoluto. Puede ser modificada a través de una acción inteligente del hombre ya que es una condición dinámica, cambiante y teóricamente controlable. Es por ello que se habla de mitigación de la vulnerabilidad y se recomiendan medidas para reducirla.

Riesgo.-Es la probabilidad de que a una población (personas, infraestructuras físicas, actividades ecconómicas, etc.) o parte de la misma, le ocurra algo nocivo o dañino. Está en función de la magnitud de la

${ }^{3}$ Lavell Alan: Op. cit., p. 32. 
amenaza y de las condiciones de vulnerabilidad de la población y/o actividades que están expuestas.

En una situación que dará resultados diferentes según el momento y condiciones en que se produzca el problema: Amenaza+Vulnerabili$\mathrm{da}=$ Nivel de riesgo.

Si sobre algunas amenazas podemos actuar, si la vulnerabilidad puede ser controlada, en consecuencia, el riesgo es el fruto de la gestión humana poco previsora sobre el territorio.

En consecuencia, se puede asegurar que cada comunidad construye: sus situaciones de riesgo, sus condiicones de supervivencia y sus costes de funcionamiento.

Algunos autores ${ }^{4}$ afirman que existe un «riesgo aceptable» entendiendo a este concepto como «el valor de probabilidad de consecuencias sociales, económicas o ambientales que, a juicio de la autoridad que regula este tipo de decisiones, es considerado como lo suficientemente bajo para permitir su uso en la planificación, la formación de requirimientos de calidad de los elementos expuestos o para fijar políticas sociales, ecocómicas y ambientales afines»...

«Esta definición que pone el énfasis en sujetos de "autoridad", asume una importancia primordial en la esfera de las organizaciones públicas y privadas» ${ }^{5}$.

Conscientes de esta aseveración, una gestión territorial que se considere responsable debe hacerse cargo de reducir el riesgo. Para ello es necesario que en cada acción de desarrollo se reflexione sobre estos temas para garantizar la supervivencia del conjunto social y el funcionamiento del sistema territorial.

\section{La responsabilidad de reducir la vulnerabilidad del territorio} y sus objetivos

Para definir cuáles son los operadores responsables, se debe clarificar primero cuál es la modalidad de gestión territorial que se ha adoptado en cada región; es decir, en qué forma se distribuye el poder en la

\footnotetext{
${ }^{4}$ Cardona Omar, D.: «Gestión ambiental y prevensión de desastres. Dos temas asociados», en Maskrey A. Los Desastres no son naturales. La RED, Tercer Mundo Editores, Bogotá, 1993.

${ }_{5}^{5}$ Lavell Alan: ...op. cit., p. 51.
} 
sociedad y el estado y cómo estos estamentos se interrelacionan. Por ejemplo: en un régimen autoritario es fácil señalar los responsables de las fallas o del éxito de determinadas acciones. No así cuando el poder está distribuido entre varios actores.

Para canalizar los programas de reducción de la vulnerabilidad es necesario conocer estrictamente quiénes son los agentes operadores en cada etapa del proceso (antes, durante la producción de un evento o después del mismo), y sus respectivas responsabilidades.

Esto ayuda a identificar las alternativas reales de reducción de la vulnerabilidad y el riesgo a desastres para cada territorio y la elaboración de planes preventivos (de manejo de los peligros) o de emergencia (planes de manejo de desastres).

En el caso de América Latina, particularmente de Argentina, se ha evolucionado en la última década hacia una forma de gestión demográfica, participativa que se desarrolla en el contexto de un proceso de globalización de la economía y de las relaciones. Nos encontramos entonces con: una administración compartida y descentralizada del territorio, múltiples actores responsables del proceso y necesidad de un activa interrelación entre los mismos.

En este contexto, la reducción de la vulnerabilidad aparece como una tarea de todos. Por supuesto las responsabilidades son diferentes, en tanto son diferentes las figuras sociales que intervienen y los instrumentos que cada uno maneja para la conducción del proceso y la organización territorial:

a) Compete el Estado

- Evaluar la vulnerabilidad de su territorio.

- Elaborar planes preventivos y planes de manejo de la emergencia.

- Promover la capacitación del conjunto social para tener una ajustada respuesta ante los problemas que resulten inevitables de enfrentar (sismos, inundaciones, huracanes, etc.).

- Buscar los recursos (humanos, materiales, financieros, etc.), para actuar y hacer más seguro el asentamiento.

- Exigir a la comunidad que participe en los programas de reducción de la vulnerabilidad.

- Incorporar en la planificación y las acciones de desarrollo, la reducción de la vulnerabilidad como elemento constitutivo del manejo ambiental. 
b) Compete al conjunto social

- Capacitarse para comprender la naturaleza de estos procesos.

- Concientizarse sobre el impacto que sus acciones pueden tener sobre su propia seguridad y subsistencia.

- Cumplir con las normas de construcción y o preventivas que atañen a la organización del territorio (permisos de construcción, materiales, normales de seguridad en edificios públicos y privados, formas de extensión de redes, etc.).

- Respetar las restricciones impuestas por las normas y de zonificación y urbanización.

- Cumplir las leyes de protección del ambiente, y si no la hubiere, impulsar su creación.

- Participar en forma activa y continua en los proyectos de preparación para la emergencia y en la creación de la normativa necesaria para aumentar su seguridad.

- Ser responsable de la seguridad de su propia familia.

- Incorporar el concepto de vulmerabilidad en todo tipo de emprendimiento socio-económico.

c) Compete a los profesionales

- Conocer la forma de evaluar la vulnerabilidad de los asentamientos humanos.

- Conocer las modalidades para reducir la vulnerabilidad y los riesgos a desastres.

- Incoporar estos elementos en sus estudios y proyectos, tanto en el sector público como privado.

- Capacitar y capacitarse para enfrentar el problema, para reducir $\mathrm{y}$, por ende, para disminuir sus costos.

La acción de estos diversos actores, converge sobre la reducción de la vulnerabilidad porque estos agentes comparten objetivos comunes:

- Aumentar la seguridad personal, social, económica y ambiental.

- Reducir la vulnerabilidad de sus instalaciones para limitar los costos de reconstrucción o rehabilitación generados por las situaciones críticas.

- Salvaguardar sus inversiones mitigando los efectos de los desastres; sobre todo de aquellos que provocan grandes pérdidas de personas y bienes. 
- Optimizar el uso de los recursos del territorio, asegurando el funcionamiento normal del mismo, aun frente a la activación de amenazas naturales.

- Racionalizar los costos de los proyectos de inversión individuales y de los de desarrollo público, reduciendo los costos no calculados que se generan por mal manejo o problemas de accidentes naturales y humanos.

- Invertir los escasos recursos disponibles en desarrollo y no en reconstrucción o rehabilitación.

- Optimizar la gestión del territorio y lograr el desarrollo sustentable del mismo.

Cómo introducir este concepto en la Gestión Territorial y por qué.

Si observamos el funcionamiento de las economías latinoamericanas, se hace evidente que el concepto de mitigación de la vulnerabilidad parece asociado estrictamente al manejo de la emergencia; pero no hay planes globales preventivos de manejo de los peligros.

Así vemos que ciudades que se destruyen, muy difícilmente cambian su estructura cuando son reconstruidas; áreas arrasadas que vuelven a construirse en el mismo lugar si que haya proyectos alternativos, etc.: «Los países de la región sólo muy lentamente toman acciones para la reducción de la vulnerabilidad o para solicitar su financiamiento; por su parte, las agencias de financiamiento para el desarrollo y los donantes, se muestran renuentes a financiarlas. La mayoría de las agencias de cooperación para el desarrollo, proporcionan pocos servcios en esta área.»

«No obstante la relación altamente favorable de costo-beneficio de las medidas preventivas, más del $90 \%$ del financiamiento internacional para el manejo de los peligros naturales en la región, se invierten en preparativos para el desastre, socorro, rehabilitación y reconstrucción, dejando menos del 10\% para la prevención antes del desaste.» ${ }^{6}$

Haciendo una simplificación y comparación un tanto cruel, podemos decir que en este tipo de situación cada comunidad está dedicando su esfuerzo a invertir en cementerios para alojar a las víctimas, en vez de crear zonas de actividades seguras para salvaguardar a la población.

${ }^{6}$ O.E.A.: «Manual sobre el manejo de peligros naturales...», op. cit., p. xi. 
No obstante, reonocer la importancia de contar con planes para el manejo de la emergencia es fundamental comprender - como ya lo plantean los organismos internacionales-que la manera más eficiente de hacer un cambio en esta forma ineficiente de administrar los territorios, es introducir la evaluación de la vulnerabilidad y las acciones de mitigación en el proceso de planificación para el desarrollo y en la formulación e instrumentación de proyectos de inversión, tanto públicos como privados.

«Todos los países para el año 2000 deberían incluir en sus planes de desarrollo sostenibles las tres metas principales del Decenio Internacional para la Reducción de los Desastres Naturales, que son:

- Exhaustivas evaluaciones nacionales de los riesgos ocasionados por amenazas naturales y la vulnerabilidad.

- Planes de mitigación y prevención a mediano y largo plazo, a nivel nacional y local incluyendo preparativos y campañas de concientización comunitaria.

- Acceso a sistemas de alarmas mundiales, regionales, nacionales y locales, además de una amplia difusión a la población de los avisos de alerta.» ${ }^{7}$

En la práctica, sin embargo, resulta a veces difícil entender insertar estos temas en la Gestión Territorial, tanto en la acción de gobierno como en la acción de los privados.

En cuanto a la primera, los organismos internacionales nos aclaran el panorama al decir que, «generalmente hablando, estas tareas pueden ser denominadas "planificación ambiental" y consisten en lo siguiente:

1. Diagnóstico de las necesidades de un área determinada.

2. Identificación de los recursos disponibles.

3. Uso de la información antedicha para la formulación de una estrategia integrada de desarrollo compuesta por varios proyectos de inversión»... «En estos procesos se usan los métodos de análisis de sistemas y de manejo de conflictos, para llegar a una distribución equitativa de costos y beneficios. Al hacerlo, se vincula la calidad de la vida humana con la calidad ambienal.» ${ }^{8}$

\footnotetext{
${ }^{7}$ Molin Valdés Helena: «Decenio Internacional para la Reducción de los Desastres Naturales (DIRDN), Oficina regional del DIRDN para América Latina y el Caribe», en Viviendo en Riesgo. La RED, FLACSO, CEPREDENAC, Colombia, 1994, p. 361.

${ }^{8}$ Bender Stephen, O.: Incorporación del manejo de peligros naturales al proceso de planificación para del desarrollo. O.E.A., op. cit., pp. 1-5.
} 
En cuanto a la segunda, es necesario concientizar a los inversores de la necesidad de incluir en sus estudios de prefectivilidad las variables Vulnerabilidad y Riesgo, como garantía de no tener que enfrentar posteriormente costos no previstos cuando sus proyectos están en plena marcha.

Para estos casos, los MOM (mapas de peligros múltiples) y los MIC (mapas de instalaciones críticas elaborados por el gobierno, pueden servir de orientación para los cálculos que deben hacer los inversores privados.

Qué instrumentos utilizar, para incorporar la redución de la vulnerabilidad en la Gestión Territorial.

En el campo de los peligros naturales, hasta ahora, como consecuencia de la falta de antecedentes sobre los fenómenos catastróficos en forma escrita era muy difícil conocer los procesos que desencadenan los desastres.

Pero hoy, con los avances de la ciencia y sobre todo de la tecnología, se dispone de múltiples instrumentos para poder analizar y evaluar el impacto de las amenazas sobre la vida de las comunidades.

«Una de las más importantes herramientas disponibles para el planificador es la percepción remota del medio ambiente. No solamente es de gran utilidad para el proceso de planificación en general, sino que es especialmente valiosa para detectar los fenómenos y producir mapas de diversos tipos de peligros naturales, cuando, como es frecuente, no existen descripciones detalladas de sus efectos»...

...«Casi todos los fenómenos naturales, geológicos, hidrológicos $\mathrm{y}$ atmosféricos, son eventos o procesos recurrentes que dejan evidencia de su anterior ocurrencia. Tal evidencia puede ser observada, analizada e integrada en el proceso de planificación.» ${ }^{9}$

Los datos recuperados por sensores remotos y por fuentes tradicionales pueden ser fácilmente procesados por los Sistemas de Información Geográficos, «para analizar estos peligros en relación con el desarrollo existente o planeado, seleccionar las formas de mitigación del

${ }^{9}$ Gaawrecki Stephen, J.: Percepción remota en la revolución dde peligros naturales. O.E.A., op. cit., pp. 4-1. 
daño que pueden causar los peligros, realzar un análisis económico de alternativas de mitigación versus ninguna mitigación, determinar el impacto de tales alternativas sobre la factibilidad económica y financiera del proyecto» ${ }^{10}$.

Estos instrumentos permiten además generar:

1. La cartografía necesaria para el manejo territorial:

M.P.M.: Los mapas de peligros múltiples tienen como finalidad presentar en una sola imagen las áreas que están sometidas a peligros que pueden desencadenar desastres a las comunicaciones que residen o que están próximas a ellas.

Estos mapas son instrumentos que pueden ser utilizados directamente en la gestión teritorial, para tomar decisiones de localización de proyectos de inversión y otras acciones de planificación, de investigación y de desarrollo, teniendo en cuenta sin embargo que éste es sólo uno de los múltiples aspectos que hay que retener para hacerlo.

M.I.C.: Los mapas de instalaciones críticas están destinados a identificar las instalaciones de uso público y privado necesarias para el desarrollo de cada asentamiento o de cada economía, que pueden ser adversamente afectadas por las amenazas naturales.

El valor de estos mapas es estratégico para preservar las que se señalan como «líneas de servicios vitales» (infraestructura y equipamientos básico, transporte), así como aquellas actividades que sostienen la economía: actividades agrícolas, industriales, etc.

2. Algunos procedimientos metodológicos para el tratamiento de la reducción de la vulnerabilidad:

En este caso, sobre todo en los ámbitos de la investigación, la disponibilidad de información clara, desagregada y confiable, permite desarrollar métodos de trabajo para captar en pequeños o grandes espacios, las diferencias de impacto de estos fenómenos y sobre todo, calcular el peso financiero que puede llegar a tener la acción humana en cada uno de ellos. En el C.E.T.E.M. (Centro dde Estrategias Territoriales para el Mercosur) se ha creado un procedimiento que permite evaluar los niveles de vulnerabilidad para guiar el crecimiento ur-

${ }^{10}$ Bello Enrique y otros: Sistemas de información geográfica en el manejo de peligros naturales. O.E.A., op. cit., pp. 5-1. 
bano y garantizar estabilidad y permanenccia del asentamiento poblacional.

Se ha perfilado para que pueda ser utilizado en los códigos de edificación y en los códigos de urbanización, como indicadores de conductas frente al medio natural y construido por el hombre. Consta de dos matrices de información:

1. La matriz de habitabilidad natural que pone en correlación la oferta del medio natural urbano y las demandas de la población. De aquí surge el I.V.N. (Indicador de Vulnerabilidad Natural Urbano).

2. La matriz de uso social del ambiente, que pone en correlación la oferta de infraestructura y servicios con la demanda del grupo social. De así el I.V.S. (Indicador de Vulnerabilidad Social Urbana).

Estas matrices han sido ensayadas en la ciudad de Mendoza (900.000 habitantes) que tiene condiciones ambientales y de vulnerabilidad muy frágiles y complejas de manejar y en la práctica ha quedado demostrado que no sólo pueden ser utilizadas por los organismos públicos, sino que pueden prestar servicios a diferentes usuarios del ámbito financiero, económico, inmobiliario, etc.

Ambas matrices dan respuestas inmediatas y confiables cuando se desea:

- Evaluar la capacidad potente del ambiente urbano.

- Evaluar la exposición de la población y sus bienes a las amenazas naturales.

- Evaluar y prever el impacto ambiental y las situaciones de riesgo potencial que producen las nuevas extensiones urbanas a nivel de ecosistema e infraestructuras básicas.

- Evaluar y prever el riesgo potencial y los costos sociales de la introducción de nuevas actividades productivas en el tejido urbano.

- Seleccionar áreas con ventajas comparativas para recibir inversiones con bajos costos ambientales y mayor seguridad frente a los desastres.

- Preparar planes y proyectos urbanos ${ }^{11}$.

${ }^{11}$ Gray de Cerdán Nelly: «I.N.V.U. Indicador de vulnerabilidad. Herramienta estratégica para la gestión socio-política de las ciudades», en Hemispheric Comngress on Disaster Reduction and Sustainables Development. Miami, Florida (USA), octubre 1966. 
También puede hacerse mención a desarrollos destinados a evaluar la vulnerabilidad en redes de transporte, como el que está desarrollando la O.E.A. con apoyo del Instituto Panamericano de Carreteras (IPC), la Oficina Humanitaria de la Comunidad Europea (ECHO) y del Instituto Superior de Ingeniería del Transporte de la Universidad Nacional de Córdoba (Argentina) ${ }^{12}$.

Es previsible que en este campo se avance rápidamente, a medida que se logre concientizar a los gestores territoriales de los beneficios de estos instrumentos.

\section{Una reflexión final}

El desarrollo de estas ideas, que impregnan los cambios y la reestructuración del mundo moderno, abren un camino extraordinario para la participación y colaboración de los Geógrafos en la Gestión del Territorio.

Los conocimientos geográficos, sus desarrollos científico-conceptuales, su tarea de docencia, su intervención profesional en este contexto, pueden considerarse hoy como parte de la estrategia de la seguridad y la subsistencia de los asentamientos humanos y configuran un expectro de tareas que son de alto valor para el desarrollo del territorio.

La formación geográfica debe encararse en consecuencia, como una tarea de formación de futuros gestores del territorio, en un sentido amplio, sin importar el lugar o la función desde la cual el Geográfo prestará sus servicios o desempeñará su trabajo o su profesión.

Estos temas son por excelencia desafíos globalizadores del conocimiento geográfico, ya que a través de ellos se puede intervenir simultáneamente en problemas de organización humana que tienen alcance local, regional, nacional e internacionla y sobre todo, permiten el desarrollo sustentable del planeta.

${ }^{12}$ Ceballos G. Arranz, P.: PerfilMap. Perfil de vulnerabilidad a Peligros Naturales. Manual del Usuario.(Versión peominar). O.E.A.-IPC-ECHO-ISIT, Washington DC., USA, 1996. 